\title{
Solar heat gain coefficient of water flow glazings
}

\author{
Pablo Sierra $^{\mathrm{a}, *}$, Juan A. Hernández ${ }^{\mathrm{a}}$ \\ ${ }^{a}$ Department of Applied Mathematics, School of Aeronautical and Space Engineering, Technical University of Madrid \\ (UPM), Plaza Cardenal Cisneros 3, 28040 Madrid, Spain
}

\section{Abstract}

Water flow glazings include a water flowing chamber which enhances their thermal behaviour. This work addresses the connection between the optical properties and the energy performance of this type of glazings. The spectral properties of each layer of the glazing determine the fraction of solar radiation absorbed in each layer, which is later transported by the water in a closed circuit. The methodology requires the optical properties retrieved from the spectral problem and the thermal behaviour is predicted following the classical double glass pane analysis detailed in EN $673(2011)$ and EN $410(2011)$. The $g$ factor and the $U$ value of this new active glazing are obtained by solving analytically this model. Besides, it is shown that a third parameter, which takes into account the temperature of the inlet water, appears to complete the thermal behaviour of the glazing. In particular, three different configurations are examined. A comparison between these configurations is conducted and the active behaviour of the glazings is explained by means of a variable $g$ factor which depends on the flow rate of the water chamber. The discussion includes some examples for the model parameters. The possibility to control dynamically the $g$ factor provides huge energy saving potentials.

Keywords: water flow glazing, $g$ factor, thermal transmittance, active behaviour, energy management, solar heat gain coefficient (SHGC)

\section{Highlights}

- A linear thermal multi-layer model is described for water flow glazings

- The solution is connected to typical thermal parameters of classical glazing

- The active behaviour of water flow glazing is shown by a variable $g$ factor

\footnotetext{
${ }^{*}$ Corresponding author

Email addresses: pablo.sierra@conectto.com (Pablo Sierra), juanantonio.hernandez@upm.es (Juan A. Hernández)
} 


\begin{tabular}{|c|c|}
\hline \multicolumn{2}{|c|}{ Nomenclature } \\
\hline$A$ & Absorptance of the glazing \\
\hline$A_{e}$ & Constant part of the secondary external heat transfer factor \\
\hline$A_{E}$ & Secondary external heat transfer factor \\
\hline$A_{i}$ & Constant part of the secondary internal heat transfer factor \\
\hline$A_{I}$ & Secondary internal heat transfer factor \\
\hline$A_{j}$ & Absorptance of the glass layer $j$ \\
\hline$A_{v}$ & Net absorptance of the water chamber \\
\hline$A_{w}$ & Absorptance of the water chamber \\
\hline$c$ & Specific heat capacity, $J / k g K$ \\
\hline$g$ & Solar heat gain coefficient (SHGC) \\
\hline$g^{O F F}$ & Solar heat gain coefficient without flow rate \\
\hline$g^{O N}$ & Solar heat gain coefficient with high flow rate \\
\hline$g_{w}$ & Flow-rate dependent contribution of the solar heat gain coefficient \\
\hline$h_{e}$ & Exterior heat transfer coefficient, $W / m^{2} K$ \\
\hline$h_{g}$ & Heat transfer coefficient of the air chamber, $W / m^{2} K$ \\
\hline$h_{i}$ & Interior heat transfer coefficient, $W / m^{2} K$ \\
\hline$h_{w}$ & Heat transfer coefficient of the water chamber, $W / m^{2} K$ \\
\hline$i_{0}$ & Normal incident solar irradiance, $W / m^{2}$ \\
\hline$k$ & Thermal conductivity, $W / m K$ \\
\hline$\dot{m}$ & Mass flow rate per unit of surface, $\mathrm{kg} / \mathrm{m}^{2} \mathrm{~s}$ \\
\hline$P$ & Net energy power of the glazing per unit of surface (water heat gain), $W / m^{2}$ \\
\hline$q$ & Total indoor heat flux (room heat gain), $W / m^{2}$ \\
\hline$q_{I}$ & Secondary indoor heat flux, $W / m^{2}$ \\
\hline$q_{j}$ & Heat flux at interface $j, W / m^{2}$ \\
\hline$R$ & Reflectance of the glazing \\
\hline$T$ & Energy transmittance of the glazing \\
\hline$U$ & Thermal transmittance of the glazing, $W / m^{2} K$ \\
\hline$U_{e}$ & Exterior thermal transmittance, $W / m^{2} K$ \\
\hline$U_{i}$ & Interior thermal transmittance, $W / m^{2} K$ \\
\hline$U_{w}$ & Thermal transmittance (water chamber-interior), $\mathrm{W} / \mathrm{m}^{2} \mathrm{~K}$ \\
\hline \multicolumn{2}{|l|}{ Greek } \\
\hline$\theta_{e}$ & Exterior temperature, $K$ \\
\hline$\theta_{i}$ & Interior temperature, $K$ \\
\hline$\theta_{I N}$ & Inlet temperature of the water chamber, $K$ \\
\hline$\theta_{j}$ & Temperature of the glass layer $j, K$ \\
\hline$\theta_{w}$ & Temperature of the water chamber, $K$ \\
\hline
\end{tabular}




\section{Introduction}

Energy management inside a building is a determining aspect in order to minimize the use of air conditioning or heating systems. To achieve this objective, the building energy efficiency can be improved using the incoming radiation of the sun, which is a natural resource.

The use of water flow glazings benefits from the large amount of energy provided by the solar radiation. Comparisons between water flow glazings and conventional single and double pane absorptive glazings were conducted in order to prove its beneficial potential application in domestic-commercial buildings with stable hot-water demands (Chow et al., 2011a). Moreover, Chow et al. (2010) studied water flow 35 glazings capabilities for cooling-demand climate. Gil-Lopez and Gimenez-Molina (2013) evaluated the efficiency of water flow glazings. Its annual performance was studied in Li and Chow (2011) through a computer simulation.

Heat exchange between the building and the exterior affects significantly energy consumption. The glazing configuration modifies the distribution of the energy absorbed in each layer of the glazing. Therefore, models are necessary to analyse thermal performances of water flow glazings. Chow et al. (2011b) proposed a model to analyse a water flow glazing composed by a water chamber between two glass panes. Advanced simulations focusing on the water chamber were studied by Lyu and Chow (2015) to evaluate different headers designs. Chow and Li (2013) analysed the impact of different glass material in the performance of the water flow glazing.

Classical glazings are usually characterized by two main values: the thermal transmittance, $U$, and the solar heat gain coefficient (SHGC), also called $g$ factor. These parameters measure the capability of the glazing to transfer heat and its capability to transfer solar energy, respectively. The $U$ value characterizes the heat transfer of a glazing regardless the edge effects and evaluates the amount of heat that passes, in steady state, through the central part of the glazing. Its determination is done according to the European Standard (EN 673, 2011). The $g$ factor is the proportion of the total solar energy which is transmitted indoors and it must be determined according to the European Standard (EN 410, 2011).

For simple glass window, Ismail and Henríquez (2003) performed a simulation to obtain the $g$ factor and the $U$ value. Besides, Gueymard and duPont (2009) studied the potential changes in solar heat gain coefficient and in $U$ value due to spectral effects. In terms of the $g$ factor, Kuhn (2014) describes a procedure to measure the solar heat gain coefficient for many different façade components.

The solar heat gain coefficient was also evaluated for more complex types of glazings, such as ventilated double windows (Carlos and Corvacho, 2015) and semi-transparent photovoltaic modules (Chen et al., 2012). Shen and Li (2016) studied the solar heat gain reduction of double glazing using cooling pipes. This configuration for the solar heat gain reduction presents some similarities with water flow glazings.

Maurer and Kuhn (2012) studied the $g$ factor of transparent façade collectors showing that the $g$ factor depends not only on the irradiation, but also on the operation of the solar collectors. This dependence makes the $g$ factor a variable parameter and, most importantly, allows to control the incoming energy. 
The behaviour of transparent façade collectors is comparable to the behaviour of water flow glazings. Hence, it is expected to find a variable and controllable $g$ factor when analysing a water flow glazings.

The applications of the $g$ factor and the $U$ value are numerous. As an example, Bansalhail et al. (1996) used them to size the various passive heating concepts for maintaining a set point temperature in a single zone building for various climatic conditions. Besides, the $g$ factor can be used in design, for instance, Kontoleon (2015) analysed the optimum glazing in terms of orientation and placement in order to maximize the solar heat gain.

70 This work is focused in defining the solar heat gain coefficient and the thermal transmittances of water flow glazings in a similar way that these parameters are defined for classical double or triple glazings in EN 673 (2011) and EN 410 (2011). There exist already a large number of software tools to perform a whole year simulation of a building in order to predict heating and cooling energy demands, but the aim of this work is to state a simple linear analytical model to define the thermal parameters of water flow glazings and to understand how it works by knowing the physical mechanisms that govern the problem. Having obtained these preliminary parameters, it is possible to predesign a building with water flow glazings. The understanding of the physical mechanisms is used to decide which is the most suitable glazing configuration for each climate. In later stages of the design procedure, a whole year simulation will quantify the energy savings validating the former elections based on this simplified model of water flow glazings.

This paper is structured as follows. The energy transfer mechanisms that are present in glazings are explained in section 2 In section 3 the hypothesis behind a linear thermal model are detailed. Section 4 revises the $g$ factor and the $U$ value of classical double glazing given by EN $673(2011)$ and EN 410 (2011). Three different configurations of water flow glazings are examined: a double glass pane with a water chamber, a triple glass pane with an air chamber and a water chamber facing outdoors and a triple glass pane with an air chamber and a water chamber facing indoors. These configurations are compared in sections 57 In section 8, universal expressions for the $g$ factor and the thermal transmittances of these three configurations are obtained. A full discussion is followed in sections 9 and 10 to understand how to select the $g$ factor and the thermal transmittance $U$ of the glazing. The active behaviour of the glazing is shown in section 11 . The dependence of the $g$ factor and the $U$ value with the flow rate allows to control the inner temperature by modifying the incoming sun energy. In these sections the physical mechanisms intervening in a water flow glazing and how these glazings are able to harvest the impinging sun energy are discussed. Finally, in section 12 examples with numerical values are discussed to enlighten the conclusions and a validation of the model is presented in section 13 The conclusions are stated in section 14 .

\section{Spectral and thermal problem}

There are three energy transfer mechanisms in water flow glazings: radiation, conduction and convection. While in glass panes and PVB layers the only mechanisms are conduction and radiation, in air or water layers, energy is also transferred by convection. 
The radiation coming from the sun impinges the glazing with visible and near infrared (NIR) components. A fraction of the radiation is absorbed in the glass panes or in the water chamber and it is later transferred to the surrounding area. Once this short wave radiation is absorbed, the glass panes or the water chamber can radiate energy with long wavelength components or far infrared (FIR) radiation associated to their temperatures. The intensity of such energy flux depends upon the temperature of the body and the nature of its surface (Lienhard IV and Lienhard V, 2008). It is also important to note that glass panes and water layers are almost transparent to short wavelengths (NIR) and opaque to long wavelengths (FIR) as it shown in Figure 1

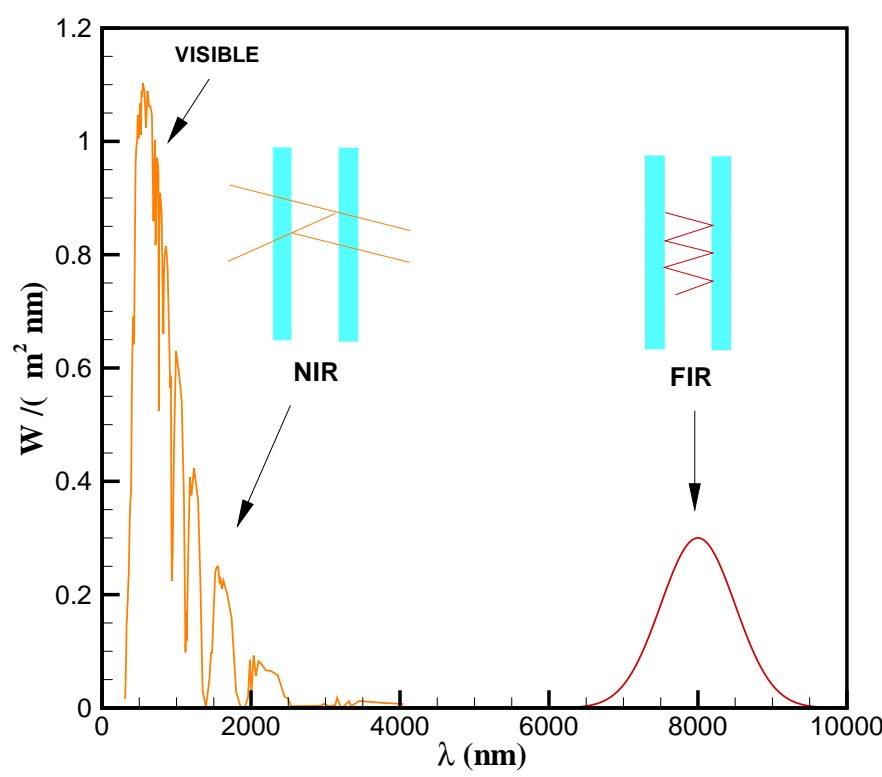

Figure 1: Spectral irradiance of solar radiation (Visible and NIR) and radiation of a thermal body (FIR).

This characteristic behaviour makes it possible to decouple short wavelengths radiation coming from the sun to long wavelength components emitted by glass panes or liquid layers. Hence, the problem of obtaining the temperatures of the different layers is split in two more simple problems: the spectral problem and the thermal problem. The spectral problem solves the solar distribution by means of multiple reflections of visible and NIR radiation and the thermal problem takes into account the transfer mechanisms: conduction, convection and FIR radiation. In the spectral problem the involved wavelengths are between $300 \mathrm{~nm}$ and $2500 \mathrm{~nm}$ (visible and NIR), which is basically the wavelength range of solar radiation at the earth surface. For the thermal problem, the transfer mechanism associated to radiation comprises long wavelengths greater than $2500 \mathrm{~nm}$ (FIR).

The incident solar energy can be transmitted, reflected or absorbed. The transmittance $(T)$, reflectance $(R)$ and absorptances for each layer $\left(A_{j}\right)$ constitute the solution of the spectral problem. These absorptances are inputs for the thermal problem.

The thermal problem consists of determining the temperatures of every layer. While in the spectral problem the multiple reflections of visible and NIR waves travel throughout the glazing, in the thermal 
problem the multiple reflections of FIR waves are bounded to an air chamber or air zones. Heat conduction results from a temperature gradient in a solid and the rate equation is Fourier's law. For liquid or gases, the heat transfer mechanism is convection, which is the combination of the heat conduction together with the transport mechanism associated to velocities of liquid or gas.

Absorption inside the glazing increases the temperature of the glazing layers and, as a result, heat is transferred indoors and outdoors. These heat fluxes are named the secondary external heat flux and the secondary internal heat flux. These secondary fluxes are a consequence of the convective thermal transfer and the FIR radiation transfer of the incident solar radiation which is absorbed by the glazing. The detailed calculations of the thermal problem are defined in the International Standard (ISO 15099, 2003).

In water flow glazings this natural convection appears in the air chamber and in the exterior and interior air boundary layers. When considering the water chamber of a water flow glazing, two mechanisms are present: forced convection originated by a circulating pump and natural convection due to density gradients inside the water chamber. Hence, it is important to note that heat transfer mechanisms inside the water chamber are challenging to model and important assumptions are required in order to have an analytical model of the water chamber. Typically, water is injected from the horizontal bottom spacer and flows vertically until it is collected by the horizontal top spacer. The flow inside the water chamber can be laminar or turbulent depending on the velocities inside the water chamber. Besides, there are regions close to the bottom and up spacers where the flow exhibits complicated patterns. However, these regions are small because the mixing process is very effective.

\section{Hypothesis to state a steady linear thermal model}

In order to simplify the mathematical model described by the International Standard (ISO 15099, 2003), some hypothesis are introduced for the different heat transfer mechanisms existing in the possible layers: water chamber, air chamber, glass panes and PVB layers.

Regarding the water chamber, it is assumed that the temperature inside the water chamber $\theta_{w}$ is uniform and the heat flux interchanged with the glass panes follows Newton's law: the convective heat flux is equal to the difference between the surface and the fluid temperatures multiplied by a heat transfer coefficient $h_{w}$. This coefficient is assumed constant once the flow rate is fixed (Li and Chow, 2011). As it was pointed out, the real flow depends on the water distribution of the spacer as well as the flow rate of the glazing and the spectral and thermal properties of the water. It is desirable to design the spacer and the water chamber to minimize gradients of temperature throughout the glazing. This study exceeds the scope of this work. However, if the spacer is designed properly, the uniform hypothesis for the water temperature is reasonable in regions not close to the spacer. It is also assumed that the inlet temperature $\theta_{I N}$ is uniform and the flow rate is a constant value $\dot{m}$. Moreover, the thermal mass of the water chamber is negligible because the characteristic time associated to glass heating or cooling is very small compared to the characteristic time in which the boundary conditions change such as sun radiation or exterior temperature. 
Regarding the air chamber, it is conceived as a closed cavity where energy transfer is due to radiation and convection. Air is transparent to short and long wavelengths and its thermal mass is negligible. Hence, the two parallel glass panes that conform the air chamber interchange heat by convection and by radiation. It is assumed that convective heat transfer inside a cavity is proportional to the difference of temperature of the parallel glass panes multiplied by a heat transfer coefficient, $h_{c}$, which is assumed as constant (EN 673 , 2011). Besides, the radiation exchange can be solved analytically. If the temperatures of the glass panes are not very different, the heat flux is proportional to the difference of temperature of the parallel glass panes multiplied by a heat transfer coefficient, $h_{r}$, which is assumed constant $(\mathrm{EN}$ 673 . 2011). Taking into account these two contributions, the heat transfer between the glass panes is proportional to the difference of temperatures multiplied by a heat transfer coefficient, $h_{g}=h_{c}+h_{r}$. Since glass panes, PVB layers or water chambers are opaque to long wavelength radiation, these wavelengths can only be transferred in air cavities, outdoors and indoors.

Regarding the single glass panes, the thermal resistance is proportional to its thickness divided by its conductivity. This thermal resistance is negligible compared to the thermal resistance associated to the heat convection mechanism. It is assumed that the temperature is uniform in space and constant in time. These hypothesis are underpinned by the following considerations: (i) the thermal resistance of the glass pane is very low and, consequently, the gradients of temperature are negligible (Freire et al. 2011), (ii) the thermal absorptance of clear or extra clear glass is low and the gradients associated to the solar absorption are negligible (Hermanns et al. 2012) and (iii) their thermal mass is negligible due to the reason mentioned for the water chamber. Hence, the temperature of glass can be considered steady or quasi steady.

Regarding the glass panes laminated with PVB layers, it is assumed that the temperature of the unit is uniform and constant in time and they will be handled as single glass panes. This is justified following the above hypothesis.

\section{Classical double glazing}

A classical double glazing consists of a double glass pane with an air chamber. Heat fluxes inside a classical double glazing are caused by the impinging sun radiation $i_{0}$ and the difference between the indoor temperature $\theta_{i}$ and the outdoor temperature $\theta_{e}$. Classical glazings are characterized by two main values: the thermal transmittance $U$ and the solar heat gain coefficient $g$. The first one, the $U$ value, characterizes the heat transfer of a glazing regardless the edge effects and evaluates the amount of heat that passes, in steady state, the central part of the glazing. It is measured in $W / \mathrm{m}^{2}$ and its determination is done according to the European Standard (EN 673, 2011). The second one, the $g$ factor, is the fraction of the total solar energy which is transmitted indoors. According to the European Standard (EN 410, 2011), the $g$ factor is the sum of the direct solar energy transmittance, $T$, and the secondary internal heat transfer factor, $A_{I}$. This secondary factor represents the fraction of the solar radiation which is absorbed in the glazing and later is transferred indoors by long wavelength radiation and by convection. The total 
indoor heat flux through the glazing, $q$, can be expressed in terms of $U$ and $g$ (ISO 18292, 2011) as:

$$
q=U\left(\theta_{e}-\theta_{i}\right)+g i_{0}
$$

where $\theta_{e}$ is the outdoor temperature and $\theta_{i}$ is the indoor temperature. For a double-glazed windows with air cavity, the thermal transmittance $U$ is defined by $($ EN 673,2011$)$ :

$$
\frac{1}{U}=\frac{1}{h_{i}}+\frac{1}{h_{e}}+\frac{1}{h_{g}},
$$

where $h_{i}$ and $h_{e}$ are the interior and the exterior heat transfer coefficients respectively and $h_{g}$ is the heat transfer coefficient of the air chamber. The $g$ factor is $($ EN 410, 2011):

$$
g=T+A_{I},
$$

being:

$$
A_{I}=U\left[A_{1}\left(\frac{1}{h_{e}}\right)+A_{2}\left(\frac{1}{h_{e}}+\frac{1}{h_{g}}\right)\right],
$$

where $A_{1}$ is the thermal absorptance of the outer glass pane, $A_{2}$ is the thermal absorptance of the inner glass pane. Once the $g$ factor and the thermal transmittance $U$ are defined for the double glazing, these two concepts are generalized in the following sections for water flow glazings.

\section{Double glazing with water chamber}

In this section it is considered the most simplest configuration for a water flow glazing: two panes of glass conforming a water chamber with a flow from bottom to top as it is shown in Figure 2 Using the simplifications presented in section 3 the energy balance for the thermal problem is presented. It is important to point out that the solution of the spectral problem should be known. The spectral problem can be solved using Optics and Window (Finlayson et al. 1993) software tools. For this configuration, $A_{1}$ denotes the absorptance of the first glass pane, $A_{w}$ the absorptance of the water chamber and $A_{2}$ the absorptance of the second glass pane. The transmittance of the glazing is denoted by $T$. All spectral properties are referred to the incoming radiation $i_{0}$. In Figure 2 the thermal profile inside each glass pane is shown constant in accordance with the hypothesis stated in section 3

The thermal mass of every layer is negligible, hence, the energy balance for each layer states that the sum of heat fluxes plus the absorbed energy must be zero. That is, for the generic layer $j$ with an absorption $A_{j}:$

$$
\sum_{k} q_{k}+A_{j} i_{0}=0
$$

where $q_{k}$ represents the heat fluxes in the boundaries of the layer (as shown in Figure 2) and $i_{0}$ is the impinging solar radiation.

For this particular configuration, the energy balance equations (5) for the three layers are:

$$
\begin{aligned}
& q_{2}=q_{1}+A_{1} i_{0}, \\
& q_{3}=q_{2}+A_{w} i_{0}+\dot{m c}\left(\theta_{I N}-\theta_{w}\right), \\
& q_{4}=q_{3}+A_{2} i_{0},
\end{aligned}
$$




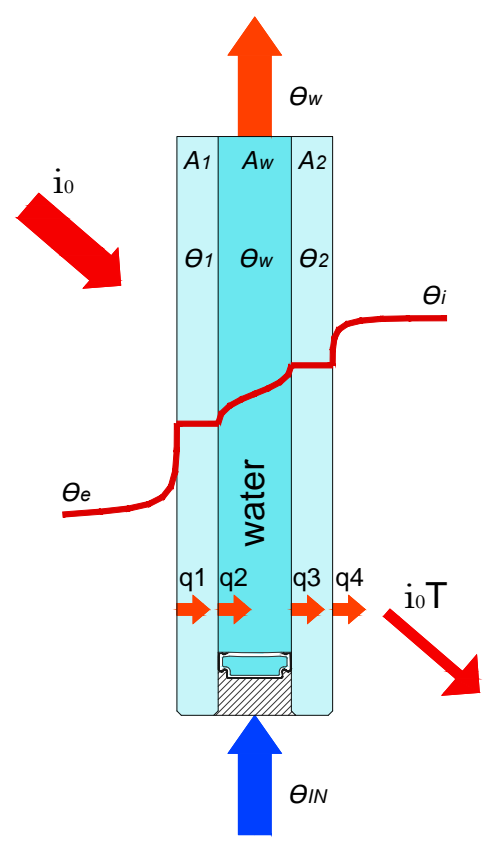

Figure 2: Thermal profile of a double glazing with water chamber. Numbering description of heat fluxes and constant temperatures of layers.

where $\theta_{I N}$ is the inlet temperature of the water chamber, $\theta_{w}$ is the temperature of the water chamber which is assumed uniform, $\dot{m}$ is the mass flow rate per unit of surface and $c$ is the specific heat capacity of the water. In equation $7 \dot{m} c\left(\theta_{I N}-\theta_{w}\right)$ represents the heat flux transported by the water chamber.

Besides, the heat fluxes in equations (6)-(8) are given by Newton's law as explained in section 3

$$
\begin{aligned}
& q_{1}=h_{e}\left(\theta_{e}-\theta_{1}\right), \\
& q_{2}=h_{w}\left(\theta_{1}-\theta_{w}\right), \\
& q_{3}=h_{w}\left(\theta_{w}-\theta_{2}\right), \\
& q_{4}=h_{i}\left(\theta_{2}-\theta_{i}\right),
\end{aligned}
$$

where $\theta_{1}$ is the temperature of the first glass pane, $\theta_{2}$ is the temperature of the second glass pane.

Linear algebraic equations (6)-(12) constitute a determined problem since there are seven equations with seven unknowns: $\theta_{1}, \theta_{2}, \theta_{w}, q_{1}, q_{2}, q_{3}, q_{4}$. It is worth noting that a boundary problem is being solved, in which $\theta_{e}$ and $\theta_{i}$ are known.

\section{Triple glazing with air and water chamber. Water facing outdoors}

In this section it is considered a triple glazing with an air chamber and a water chamber facing outdoors as 
for the heat fluxes by means of Newton's law, the following set of equations is obtained maintaining the same notation:

$$
\begin{aligned}
& q_{1}=h_{e}\left(\theta_{e}-\theta_{1}\right) \\
& q_{2}=q_{1}+A_{1} i_{0}, \\
& q_{2}=h_{w}\left(\theta_{1}-\theta_{w}\right) \\
& q_{3}=q_{2}+A_{w} i_{0}+\dot{m c}\left(\theta_{I N}-\theta_{w}\right) \\
& q_{3}=h_{w}\left(\theta_{w}-\theta_{2}\right) \\
& q_{4}=q_{3}+A_{2} i_{0}, \\
& q_{4}=h_{g}\left(\theta_{2}-\theta_{3}\right) \\
& q_{5}=q_{4} \\
& q_{6}=h_{i}\left(\theta_{3}-\theta_{i}\right), \\
& q_{6}=q_{5}+A_{3} i_{0} .
\end{aligned}
$$

Note that the air chamber has no absorption. Therefore, the energy balance for this layer states that the inlet heat flux is equal to the outlet heat flux as seen in equation 20]. Equations 13 - 22 constitute a set of ten linear algebraic equations for the ten unknowns: $\theta_{1}, \theta_{2}, \theta_{3}, \theta_{w}, q_{1}, q_{2}, q_{3}, q_{4}, q_{5}, q_{6}$. Hence, the thermal problem is determined for this glazing.

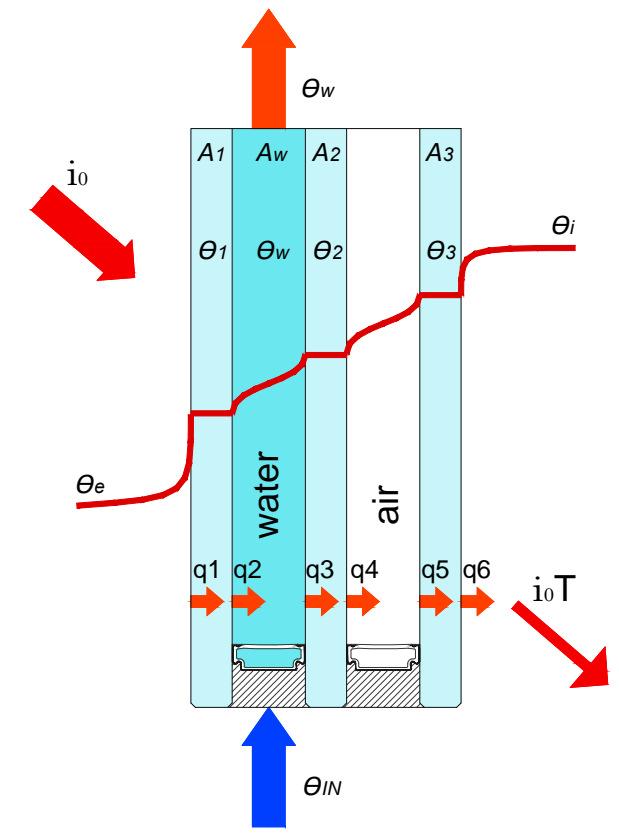

Figure 3: Thermal profile of a triple glazing with an air chamber and a water chamber facing indoors. Numbering description of heat fluxes and constant temperatures of layers. 


\section{Triple glazing with air and water chamber. Water facing indoors}

In this section it is considered a variant of the preceding triple glazing. In this configuration the water chamber is facing indoors as it is shown in Figure 4 The idea behind this configuration is to compare the $g$ factor and the efficiency of this configuration with the preceding ones in order to determine which of these configurations is more suitable for the weather conditions and the energy constraints of the project. Imposing energy balance for each layer and the definitions of heat fluxes by means of Newton's law, the following set of equations is obtained maintaining the same notation:

$$
\begin{aligned}
& q_{1}=h_{e}\left(\theta_{e}-\theta_{1}\right), \\
& q_{2}=q_{1}+A_{1} i_{0}, \\
& q_{2}=h_{g}\left(\theta_{1}-\theta_{2}\right), \\
& q_{3}=q_{2}, \\
& q_{4}=h_{w}\left(\theta_{2}-\theta_{w}\right), \\
& q_{4}=q_{3}+A_{2} i_{0}, \\
& q_{5}=h_{w}\left(\theta_{w}-\theta_{3}\right), \\
& q_{5}=q_{4}+A_{w} i_{0}+\dot{m} c\left(\theta_{I N}-\theta_{w}\right), \\
& q_{6}=h_{i}\left(\theta_{3}-\theta_{i}\right), \\
& q_{6}=q_{5}+A_{3} i_{0} .
\end{aligned}
$$

Once again, equations (23)- 32 constitute a set of ten linear algebraic equations with ten unknowns.

The solution of these set of equations determines the thermal profile and the heat fluxes in the glazing.

\section{Thermal performance of water flow glazings}

The objective of this section to provide with an expression for the secondary internal heat flux $q_{I}$ of the glazing configurations described in sections 57 This secondary internal heat flux $q_{I}$ corresponds to $q_{4}$ in the double glazings with a water chamber (section 5 ) and to $q_{6}$ in the triple glazings with water and air chamber (sections 6 and 7). Hence, $q_{I}$ is obtained by solving the sets of equations presented in sections 517 for each glazing configuration. A general solution of theses sets of equations, valid for all glazing configuration, is presented below:

$$
q_{I}=U\left(\theta_{e}-\theta_{i}\right)+U_{w}\left(\theta_{I N}-\theta_{i}\right)+A_{I} i_{0}
$$

where

$$
\begin{aligned}
U & =\frac{U_{i} U_{e}}{\dot{m} c+U_{e}+U_{i}}, \\
U_{w} & =\frac{U_{i} \dot{m} c}{U_{i}+U_{e}+\dot{m} c}, \\
A_{I} & =\left(\frac{U_{i}}{\dot{m} c+U_{e}+U_{i}}\right) A_{v}+A_{i} .
\end{aligned}
$$




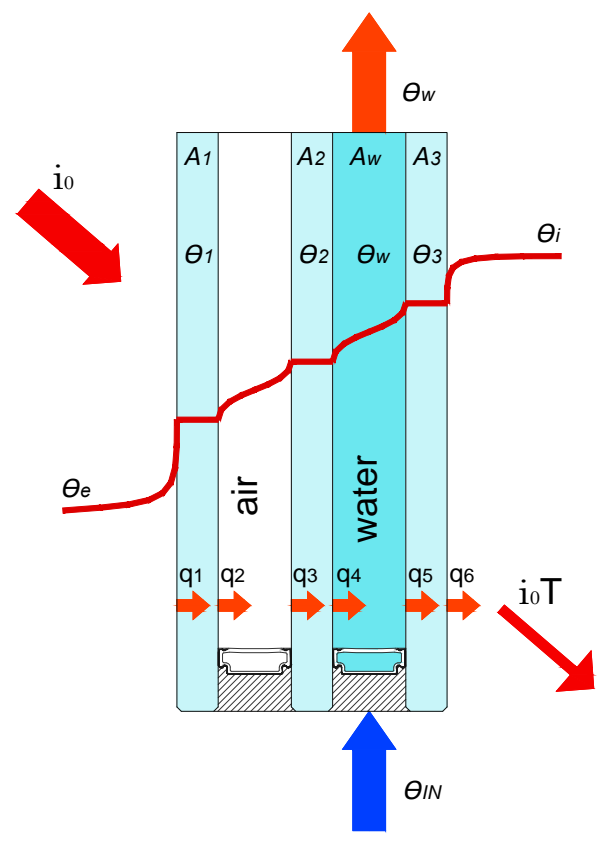

Figure 4: Thermal profile of a triple glazing with an air chamber and a water chamber facing indoors. Numbering description of heat fluxes and constant temperatures of layers.

$U_{i}, U_{e}$ are thermal transmittances which can be obtained by means of the convective heat coefficients $h_{e}, h_{i}, h_{g}, h_{w}$ and are given in Table 1 for the preceding three different glazings. To understand the physical meaning of $U_{i}$ and $U_{e}$, their definitions given by Table 1 can be useful. For all configurations the thermal resistance $1 / U_{e}$ is the sum of the thermal resistances from the water chamber to outdoors. Similarly, the thermal resistance $1 / U_{i}$ is the sum of the thermal resistances from the water chamber to indoors. Hence, $U_{e}$ can be understood as the thermal transmittance between the water chamber and outdoors and $U_{i}$ as the thermal transmittance between that water chamber and indoors. $\dot{m} c$ is the capacity of the water flow to transfer energy.

The secondary internal heat transfer factor $A_{I}$ depends on the net absorptance of the water chamber $A_{v}$, given in Table 2, and on the constant part of the secondary internal heat transfer factor $A_{i}$, given in Table 3 As may be seen, the solution of the thermal problem depends on the model parameters $h_{e}, h_{i}, h_{w}, h_{g}$, the absorptances of different layers $A_{j}$ and the boundary conditions $i_{0}, \theta_{e}, \theta_{i}, \theta_{I N}, \dot{m} c$.

Furthermore, the total indoor heat flux $q$ is the sum of transmitted solar energy radiation $T i_{0}$ associated to the spectral problem and the secondary internal heat flux associated to the thermal problem $q_{I}$ :

$$
q=T i_{0}+q_{I} .
$$

Taking into account equations (33) and (37), a universal solution for the total indoor heat flux $q$ is yielded:

$$
q=U\left(\theta_{e}-\theta_{i}\right)+U_{w}\left(\theta_{I N}-\theta_{i}\right)+g i_{0}
$$


where the $g$ factor is:

$$
g=T+A_{I}
$$

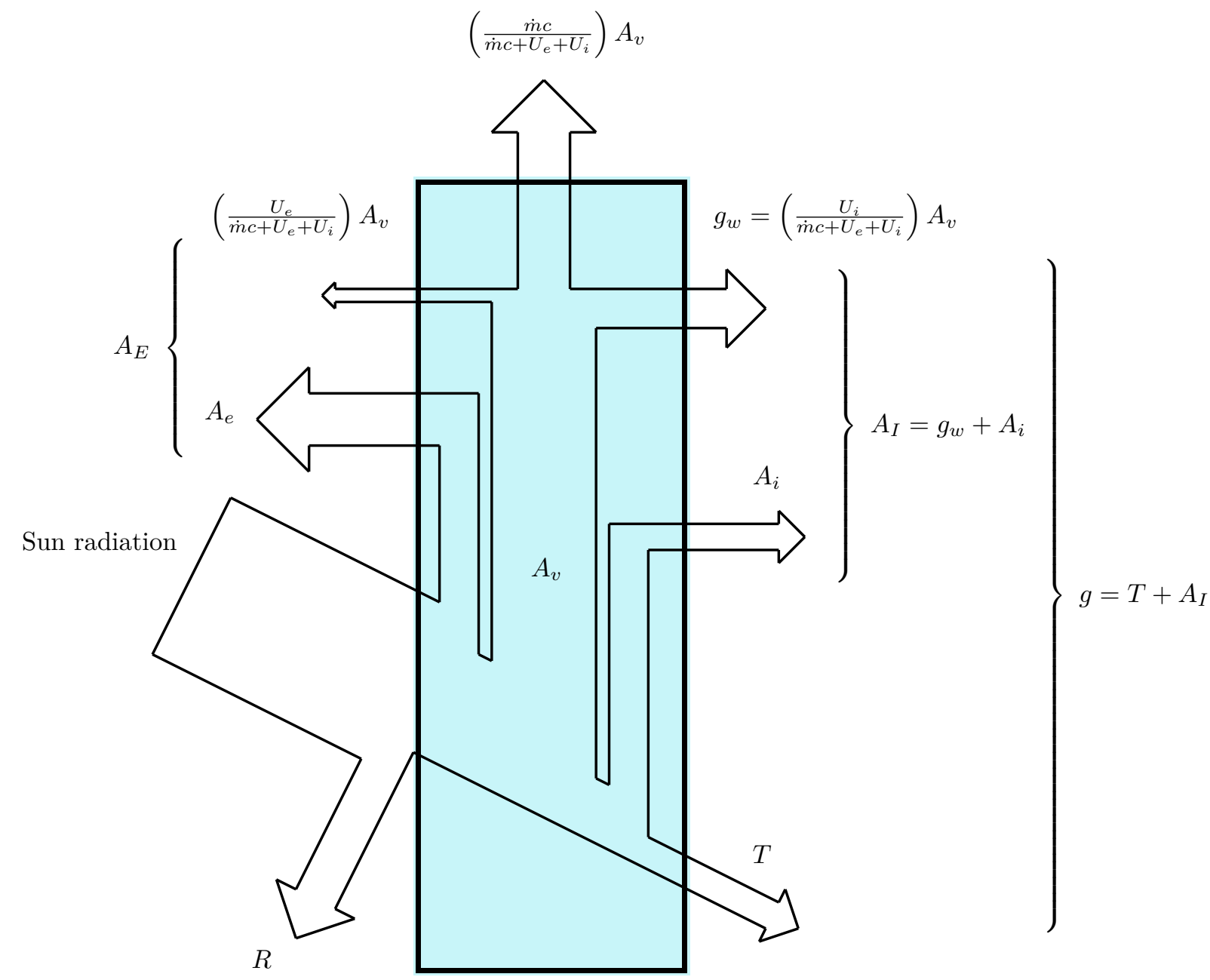

Figure 5: Distribution of the impinging sun radiation when strikes the glazing, which is divided in: the energy transmittance $(T)$, the reflectance $(R)$, the constant part of the secondary external and internal heat transfer factor $\left(A_{e}\right.$ and $A_{i}$ respectively) and the net absorptance of the water chamber $\left(A_{v}\right)$.

Figure 5 represents how the impinging sun radiation is divided into different contributions in order to provide a comprehensive description of the $g$ factor. The absorbed energy from the impinging sun radiation can be divided into three contributions: a fraction of the radiation is reflected (reflectance, $R$ ), a fraction is transmitted (transmittance, $T$ ) and the remaining fraction is absorbed (absorptance, $A$ ). 


\begin{tabular}{|c|c|c|}
\hline Glazing & $U_{e}$ & $U_{i}$ \\
\hline Water chamber & $\frac{1}{U_{e}}=\frac{1}{h_{e}}+\frac{1}{h_{w}}$ & $\frac{1}{U_{i}}=\frac{1}{h_{i}}+\frac{1}{h_{w}}$ \\
\hline $\begin{array}{c}\text { Water chamber } \\
\text { facing outdoors }\end{array}$ & $\frac{1}{U_{e}}=\frac{1}{h_{e}}+\frac{1}{h_{w}}$ & $\frac{1}{U_{i}}=\frac{1}{h_{i}}+\frac{1}{h_{g}}+\frac{1}{h_{w}}$ \\
\hline $\begin{array}{c}\text { Water chamber } \\
\text { facing indoors }\end{array}$ & $\frac{1}{U_{e}}=\frac{1}{h_{e}}+\frac{1}{h_{g}}+\frac{1}{h_{w}}$ & $\frac{1}{U_{i}}=\frac{1}{h_{i}}+\frac{1}{h_{w}}$ \\
\hline
\end{tabular}

Table 1: Thermal transmittances $U_{e}$ and $U_{i}$ for the three different water flow glazings.

This conservation is expressed as:

$$
1=R+T+A
$$

The absorbed energy $(A)$ can be further split in three different contributions: a fraction (constant part of the secondary internal heat transfer factor, $A_{i}$ ) is transferred indoors independently of the flow rate, a fraction (constant part of the secondary external heat transfer factor, $A_{e}$ ) is transferred outdoors independently of the flow rate and the remaining fraction (net absorptance of the water chamber, $A_{v}$ ) is transferred indoors, outdoors and transported by the water chamber depending on the flow rate. These balance yields:

$$
A=A_{e}+A_{v}+A_{i} .
$$

For each configuration, the expressions for the constant part of the secondary external heat transfer factor $\left(A_{e}\right)$ are gathered in Table 3

The contribution $A_{v}$ can be divided, again, into three different contributions that are indicated in Figure 5 Keeping the physical meanings of $U_{e}, U_{i}$ and $\dot{m} c$ in mind, these contributions can be interpreted in the following way. For a given flow rate, $A_{v} U_{e} /\left(\dot{m} c+U_{e}+U_{i}\right)$ is the proportion of the total absorbed energy which is transferred outdoors, $A_{v} \dot{m} c /\left(\dot{m} c+U_{e}+U_{i}\right)$ is the proportion transported by the water chamber and the third contribution, called $g_{w}$, is the proportion transferred indoors:

$$
g_{w}=\left(\frac{U_{i}}{\dot{m} c+U_{e}+U_{i}}\right) A_{v} .
$$

This distribution is determined by the ratios between $U_{i}, U_{e}$ and $\dot{m} c$.

Once all the contributions are identified, the two secondary heat transfer factors are defined: the secondary external heat transfer factor $A_{E}$ and the secondary internal heat transfer factor $A_{I}$. These two factors include a constant and a flow-rate dependent contribution. $A_{E}$ is defined as:

$$
A_{E}=\left(\frac{U_{e}}{\dot{m} c+U_{e}+U_{i}}\right) A_{v}+A_{e}
$$

while $A_{I}=A_{i}+g_{w}$ is defined in equation (36). The $g$ factor, which is the net proportion of sun radiation $i_{0}$ that passes indoors, is given by equation (39). This equation can be rewritten using equation (34) and 
the definition of $g_{w}$ given by equation 42 , yielding:

$$
g=T+A_{i}+g_{w} .
$$

\begin{tabular}{|c|c|}
\hline Glazing & $A_{v}$ \\
\hline Water chamber & $A_{1}\left(\frac{U_{e}}{h_{e}}\right)+A_{2}\left(\frac{U_{i}}{h_{i}}\right)+A_{w}$ \\
\hline $\begin{array}{l}\text { Water chamber } \\
\text { facing outdoors }\end{array}$ & $A_{1}\left(\frac{U_{e}}{h_{e}}\right)+A_{2}\left(\frac{1}{h_{g}}+\frac{1}{h_{i}}\right) U_{i}+A_{3}\left(\frac{U_{i}}{h_{i}}\right)+A_{w}$ \\
\hline $\begin{array}{l}\text { Water chamber } \\
\text { facing indoors }\end{array}$ & $A_{1}\left(\frac{U_{e}}{h_{e}}\right)+A_{2}\left(\frac{1}{h_{g}}+\frac{1}{h_{e}}\right) U_{e}+A_{3}\left(\frac{U_{i}}{h_{i}}\right)+A_{w}$ \\
\hline
\end{tabular}

Table 2: Absorptance $A_{v}$ for the three different water flow glazings.

\begin{tabular}{|c|c|c|}
\hline Glazing & $A_{i}$ & $A_{e}$ \\
\hline Water chamber & $A_{2}\left(1-\frac{U_{i}}{h_{i}}\right)$ & $A_{1}\left(1-\frac{U_{e}}{h_{e}}\right)$ \\
\hline $\begin{array}{l}\text { Water chamber } \\
\text { facing outdoors }\end{array}$ & $A_{2}\left[1-\left(\frac{1}{h_{g}}+\frac{1}{h_{i}}\right) U_{i}\right]+A_{3}\left(1-\frac{U_{i}}{h_{i}}\right)$ & $A_{3}\left(1-\frac{U_{i}}{h_{i}}\right)$ \\
\hline $\begin{array}{c}\text { Water chamber } \\
\text { facing indoors }\end{array}$ & $A_{3}\left(1-\frac{U_{i}}{h_{i}}\right)$ & $A_{1}\left(1-\frac{U_{e}}{h_{e}}\right)+A_{2}\left[1-\left(\frac{1}{h_{g}}+\frac{1}{h_{e}}\right) U_{e}\right]$ \\
\hline
\end{tabular}

Table 3: Absorptances $A_{i}$ and $A_{e}$ for the three different water flow glazings.

From equations (34)-35) it can be easily observed that the thermal transmittances $U$ and $U_{w}$ depend on the flow rate $\dot{m}$. It can also be noted from equation (44) that the $g$ factor depends on the flow rate through $g_{w}$, which is the flow-rate dependent contribution of the solar heat gain coefficient.

Comparing equations (34)-39) with their equivalent for a classical double glazing, equations (1)-(4), two main differences are identified: (i) water flow glazings have the potential to control or to modify the thermal performance by means of the flow rate $\dot{m}$ and (ii) a new thermal transmittance $U_{w}$ appears which takes into account that the temperature of the innermost glass pane is close to the inlet temperature $\theta_{I N}$. It is important to note that in classical double glazings the $g$ factor and the $U$ value are constant and, consequently, the glazing behaves statically not being able to adapt to different climatic conditions. However, the active character of water flow glazings permits to modify their thermal performance using the flow rate $\dot{m}$. Hence, a variation in the flow rate of the water chamber allows to modify the thermal 
transmittances $U$ and $U_{w}$, as well as the $g$ factor of the glazing. This allows to manage the incoming sun energy.

Next section follows a detailed discussion to understand how to manage the energy and how to select the proper glazing which fits best with our purposes.

\section{Solar heat gain coefficient}

The definition of the solar heat gain coefficient given in equation (3) for a water flow glazing yields using equation 44:

$$
g=\left(\frac{U_{i}}{\dot{m} c+U_{e}+U_{i}}\right) A_{v}+A_{i}+T
$$

There are two clearly differentiated contributions in the solar heat gain coefficient for a water flow glazing. The first contribution is associated to the two first terms on the RHS of equation 45 and corresponds to the absorbed energy by the different glass panes, PVB layers and water layers. This absorbed energy is transferred indoors by means of convection mechanisms and FIR emissions. In fact, this term is the secondary internal heat transfer factor $\left(A_{I}\right)$, as given in equation (36). The second contribution is associated to the direct solar energy transmittance $(T)$. This last term is related to the visible and NIR wavelengths.

If the flow rate $\dot{m}$ is high enough, i.e. $\dot{m} c \gg U_{e}+U_{i}$, the contribution to the $g$ factor of the first term is negligible following equation (45). Consequently, the first term is associated to the absorbed energy which is able to transport the water chamber. On the contrary, the second term of equation (45) cannot be modified by varying the flow rate $\dot{m}$. This second term is associated to the absorbed energy which is transferred indoors. Similarly, the third term which is the energy transmittance (a spectral property) of the glazing cannot be controlled by varying the flow rate.

Depending on the energy demands of the building, the proper water flow glazing should be designed. For that reason, a deep understanding of the spectral and thermal parameters of each layer is required to define a composition.

\subsection{Flow-rate dependent contribution of the solar heat gain coefficient}

Considering the water chamber as an open system, the power per unit of surface which can transport is:

$$
P=\dot{m} c\left(\theta_{w}-\theta_{I N}\right) .
$$

The unknown $\theta_{w}$ is obtained by solving the equations presented in sections 57 To obtain the water temperature of the configuration presented in section 5 equations $(9)$ and $(10)$ are divided by the heat transfer coefficient of each equation. Then, these two equations are added and equation (6) is substituted in the resulting equation. The same procedure is repeated with equations 111,12 and (8). Finally, these two resulting equations are taken to equation (7) to clear up $\theta_{w}$. For configurations of sections 647 a similar procedure is carried out. Thus, the temperature of the water chamber is yielded:

$$
\theta_{w}=\frac{A_{v} i_{0}+U_{i} \theta_{i}+U_{e} \theta_{e}+\dot{m} c \theta_{I N}}{\dot{m} c+U_{e}+U_{i}} .
$$


This temperature is then substituted in equation (46) to reach an expression for the power as a function of the boundary conditions and the thermal parameters:

$$
P=\left(\frac{\dot{m} c}{\dot{m} c+U_{e}+U_{i}}\right)\left[A_{v} i_{0}+U_{i}\left(\theta_{i}-\theta_{I N}\right)+U_{e}\left(\theta_{e}-\theta_{I N}\right)\right]
$$

$A_{v} i_{0}$ represents the energy absorbed by the water chamber, plus the energy transferred by convection from the glass panes due to the glass panes absorption. Note that in equation $48, A_{v} i_{0}$ is weighed by parameters or $(i i)$ choosing the spectral absorptances of each layer.

The first way to maximize $A_{v}$ is to modify the weighting factors by means of the thermal parameters. Since $U_{i}$ and $U_{e}$ are referred to the water chamber, the weighting factor of the water absorptance in Table 2 is the unity. The remaining weighting factors are the ratios between the thermal transmittances. by the thermal transmittance between this layer and outdoors. Similarly, when the layer is to the left, the ratio is the thermal transmittance $U_{i}$ divided by the thermal transmittance between this layer and indoors. To enlighten this analysis, a double glazing with a water chamber is considered. If the water chamber is very efficient with $h_{w}$ high enough, then $U_{i}$ tends to $h_{i}$ and $U_{e}$ tends to $h_{e}$ (see Table 1). 
Hence $A_{v}=A_{1}+A_{w}+A_{2}$, which means that the water chamber can transport all the absorbed energy because the convective mechanism between the water and the glass panes is so efficient that all the heat is available to be transported, although the heat is absorbed by the glass panes instead of by the water chamber.

The second way to maximize $A_{v}$ is to choose the spectral absorptances of each layer for given thermal parameters and for constant absorptance of the glazing $A$. The net absorptance of the water chamber $A_{v}$ is a weighted averaged expression of different glass spectral absorptances $A_{j}$ and $A_{w}$ (Table 2). Since each absorptance $A_{j}$ is weighted with a value less than one except the water absorptance $A_{w}$ which is weighted with the unity, the spectral configuration which maximizes $A_{v}$ is the one with extra clear glass panes, i.e. $A_{j}=0$, in which the water chamber is the only layer that absorbs sun energy. In that case, $A_{v}=A_{w}$ and

$$
g=\left(\frac{U_{i}}{\dot{m} c+U_{e}+U_{i}}\right) A_{w}+T .
$$

To obtain a desired level of absorptance in the water layer, the only possibility is to mix the water with NIR and visible filter additives to obtain the required value of $A_{w}$. To further increase the absorptance, filter additives could be added to decrease transmittance in the visible spectra.

It was shown that maximizing $A_{v}$ requires $A_{j}=0$. However, this requirement cannot be fulfilled as the value of the absorptance for each layer differs from zero. The lowest absorptance values can be obtained using extraclear glass. Even in that case absorptances are important. Therefore, the most suitable distribution of $A_{j}$ needs to be identified. If a triple glazing with the water chamber facing indoors is considered, the best configuration is an extraclear glass pane facing outdoors, an extraclear glass pane facing indoors and a black glass pane in the middle. The thermal resistance of the air chamber reduces the energy losses and, therefore, $A_{v}$ is increased. In a triple glazing with the water chamber facing outdoors, the best choice is selecting an extraclear glass pane facing outdoors, an extraclear glass pane facing indoors and a black glass pane in the middle.

\subsection{Constant part of the secondary internal heat transfer factor}

The second term of equation (45), $A_{i}$, is related to the radiation absorbed to the right of the water chamber and later is directly transferred indoors by means of convection and far infrared emissions.

The absorbed heat of every layer is proportional to its absorptance $A_{j}$. If this layer is facing indoors or it is separated by an air chamber, some part of this heat is transferred indoors. The other part is transferred to the water chamber and it is included in the term $A_{v}$. Tables 2 and 3 show that the factor that weighed an absorptance in the calculation of $A_{i}$ plus the factor that weighed the same absorptance in the calculation of $A_{v}$ is equal to the unit.

Since $A_{i}$ does not depend on the flow rate, this term can not be used to vary the $g$ factor by modifying the flow rate. Hence, to have the maximum range of different $g$ values, this term should be nullified. Looking at the expression for $A_{i}$ from Table 3 it is shown that if the water chamber is facing indoors, $A_{i}$ becomes zero as $U_{i}=h_{i}$. Since $U_{i}$ is the thermal transmittance between the water layer and indoors, and 
$h_{i}$ is the thermal transmittance between the innermost glass pane and indoors, $U_{i}$ is approximately $h_{i}$ if the convective coefficient of the water chamber $h_{w}$ is high enough. This can be seen from expressions of Table 1

However, when the water chamber is facing outdoors, $A_{i}$ cannot be nullified even with high values of $h_{w}$. If $h_{w}$ is high enough, $1 / U_{i} \approx 1 / h_{i}+1 / h_{g}$ from Table 1 and the thermal transmittance $U_{i}$ is smaller than the thermal transmittance $h_{i}$ due to the thermal resistance of the air chamber $\left(1 / h_{g}\right)$. Hence, $A_{i}$ for a water chamber facing outdoors with high values of $h_{w}$ becomes:

$$
A_{i}=A_{3}\left(1-\frac{U_{i}}{h_{i}}\right) .
$$

This equation shows that the absorbed energy by the innermost glass pane (layer 3) can not be transported by the water chamber due to presence of an air chamber with high thermal resistance between the water chamber and the layer 3 .

\section{Thermal transmittances of the glazing}

The secondary internal heat flux $q_{I}$ given by equation $(33)$ states that there are two contributions to the heat flux which is transferred indoors: (i) the energy associated to the absorbed sun radiation in the glazing which has been analysed in section 9 and (ii) the energy associated to the differences of temperatures $\theta_{w}, \theta_{i}$ and $\theta_{e}$. The thermal transmittance $U$ given by equation (34) measures the heat looses or gains associated to the difference of temperatures $\theta_{e}-\theta_{i}$. Besides, there is another thermal transmittance $U_{w}$ given by equation (35) which measures the heat looses or gains associated to the difference of temperatures $\theta_{I N}-\theta_{i}$.

It can be seen from their definitions in equations (34) and 35 that these transmittances depend on the flow rate $\dot{m}$. If the flow rate $\dot{m}$ is high enough, the $U$ value becomes zero and $U_{w}$ approaches to $U_{i}$. That is, the building does not interchange heat with outside because an isothermal envelope of temperature $\theta_{I N}$ is created and the heat losses or gains are caused by the difference $\theta_{I N}-\theta_{i}$.

If the system is stopped $(\dot{m}=0)$, then $U$ and $U_{w}$ become:

$$
\begin{aligned}
U & =\frac{U_{i} U_{e}}{U_{e}+U_{i}}, \\
U_{w} & =0 .
\end{aligned}
$$

Hence, it is the same $U$ value that appeared in classical glazings seen in equation (2).

\section{Active behaviour of the glazing}

The $g$ factor and the thermal transmittances change depending on the flow rate. This fact provides an active behaviour of the glazing. An active behaviour is the dynamic capacity to adapt to the environment depending on weather conditions. To understand the range of different values for the $g$ factor and the 
thermal transmittances $U$ and $U_{w}$, upper and lower bounds of these values are obtained. These bounds are obtained when the system is stopped and they are named with the superscript $O F F$ and when the system is at a very high flow rate and the corresponding values are denoted with the superscript $O N$.

If the system is stopped $(\dot{m}=0)$, the total heat flux in equation 38 becomes:

$$
q=U^{O F F}\left(\theta_{e}-\theta_{i}\right)+g^{O F F} i_{0},
$$

where

$$
U^{O F F}=\frac{U_{i} U_{e}}{U_{e}+U_{i}}, \quad g^{O F F}=\left(\frac{U_{i}}{U_{e}+U_{i}}\right) A_{v}+A_{i}+T .
$$

In this case, the glazing behaves as a classical double pane glazing and the thermal transmittance given by 55 coincides with the classical definition for the $U$ value as seen in section $10 g^{O F F}$ represents the proportion of the sun radiation which is transferred indoors when the system is stopped.

If the flow rate is such that $\dot{m} c \gg U_{e}+U_{i}$, the total heat flux of equation 38 becomes:

$$
q=U_{w}^{O N}\left(\theta_{I N}-\theta_{i}\right)+g^{O N} i_{0}
$$

where

$$
U_{w}^{O N}=U_{i}, \quad g^{O N}=A_{i}+T
$$

That is, the building does not interchange heat with outside because an isothermal envelope at temperature $\theta_{I N}$ is created and the heat losses or gains are due to the difference $\theta_{I N}-\theta_{i}$. As it was pointed out in section 9.2 the term $A_{i}$ is associated to absorbed energy which is transferred indoors by FIR radiation and by convection mechanisms. Generally, this term is undesirable and to eliminate it one must choose non absorbing PVB layers or glass panes to the right of the water chamber.

The $g^{O F F}$ value is the upper value of $g$ and $g^{O N}$ is the lower. Hence, the maximum variation of the $g$ factor from equations 55 and (57) is:

$$
g^{O F F}-g^{O N}=\left(\frac{U_{i}}{U_{e}+U_{i}}\right) A_{v}
$$

If the water flow glazing is used to manage the incoming solar energy entering into the building, a maximum range of different values of the $g$ factor is required. Therefore, it is important to analyse which glazing configuration maximizes the range of $g$ values.

If the water flow glazing is considered symmetrical, i.e. $U_{e}=U_{i}$, the maximum variation of the $g$ values is $A_{v} / 2$. Hence, it seems that symmetrical glazings are not desirable because their potential range of the $g$ value is divided by two.

Looking at expression (58), to maximize the range of values of the $g$ factor, the $U_{i}$ value should be much higher than the $U_{e}$ which is equivalent to diminish the thermal resistance from the water chamber to indoors and at the same time increase the thermal resistance from the water chamber to outdoors. It can be accomplished by means of an air chamber that reduces the heat flux from the water chamber to outdoors. This is the glazing configuration selected in section 7. On the contrary, the glazing configuration of section 6 has the $U_{i}$ value much smaller than the $U_{e}$ value and, consequently, the variation of the $g$ factor is small. 


\section{Numerical examples}

In this section some numerical examples are analysed to enlighten the active behaviour discussed in created, Window can calculate averaged solar absorptances for each layer at different angles of incidence. Finally, these absorptances can be exported to other programs to calculate the thermal problem.

It is important to note that Window models each of the layers separated by a gas gap, i.e. the layers are not in contact. Hence, the normal procedure would consist of creating laminates with Optics and then import them in Window. One problem of this approach is that Window can not separate the different layers of a laminate. Hence, Window calculates the net absorptance of a laminate as a block without distinguishing between panes or PVB layers. This approach is valid for laminates since the thermal model presented in this work takes into account the net absorptance of the complete laminate. However, when considering blocks of glass-water-glass, this approach is not applicable because the thermal model needs the specific water absorptance.

To overcome this problem, special isolated layers of glass and water were created in Optics and then imported in Window. The files imported by Optics have a special format. They are composed by four columns: wavelength, thermal transmittance, reflectance and back reflectance of the specimen. These values can be obtained experimentally or calculated from the index of refraction and coefficient 
of extinction of the specimen. For example, since a water layer is surrounded by glass, it is necessary to account for the glass-water and water-glass interfaces when generating the special water layer. The reflectivity of these interfaces is governed by the index of refraction of glass and the index of refraction of water which are known as a function of the wavelength. The same applies for a glass layer surrounded by water and air. Interfaces of air-glass and glass-water were considered when generating this special layer. Once these special layers were created in Optics, they were imported in Window and a glazing system was composed with these special layers. Later, the absorptances for each layer (glass, water, glass) could be determined using Window. This procedure can be extended to more complex glazing arrangements. The resulting values of absorptances are summarized in Table 4

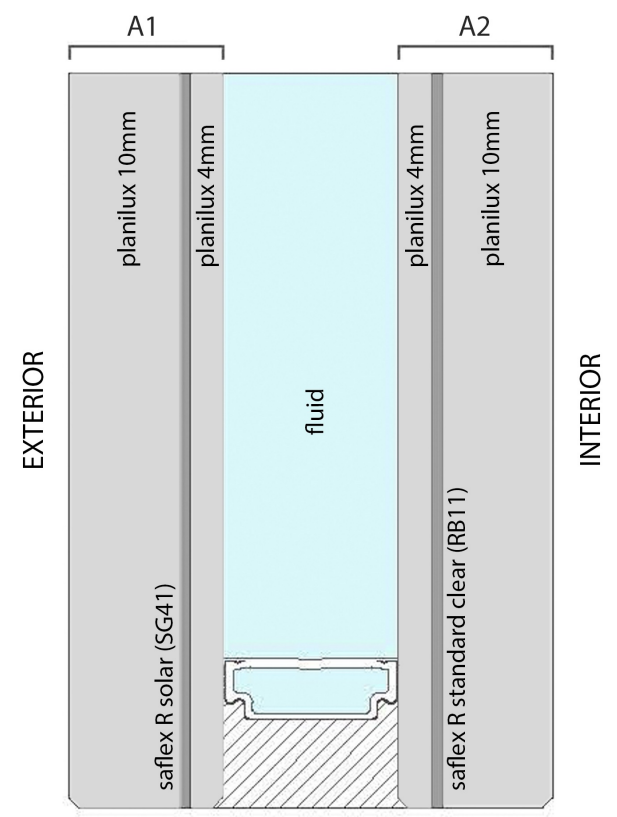

Figure 6: Double glazing with water chamber configuration.

With respect to the thermal problem, the coefficients $h_{i}$ and $h_{e}$ are taken from the European Standard (EN 673, 2011), being $h_{i}=8 \mathrm{~W} / \mathrm{m}^{2} \mathrm{~K}$ and $h_{e}=23 \mathrm{~W} / \mathrm{m}^{2} \mathrm{~K}$ for common vertical glazings. A typical value for the heat transfer coefficient of the water chamber, $h_{w}$, is $100 \mathrm{~W} / \mathrm{m}^{2} K$ (Baehr and Stephan, 2006). According to the European Standard (EN 673, 2011) for vertical glazings, $h_{c}=1.16 \mathrm{~W} / \mathrm{m}^{2} \mathrm{~K}$ being argon the gas in the air chamber and performing the calculations with the limit standard conditions established in this Standard. The heat transfer coefficient due to radiation, $h_{r}$, can be neglected because the air chamber emissivity is very low due to the water chamber. Therefore, the heat transfer coefficient of the water chamber, $h_{g}=h_{c}+h_{r}$, is $1.16 \mathrm{~W} / \mathrm{m}^{2} K$. Since the fluid inside the chamber is a mixture of water and glycol, the value of the specific heat capacity has been taken as $c=3600 \mathrm{~J} / \mathrm{kgK}$.

From equations (55) and (57) and with the spectral properties given in Table 4 and the thermal values given above, the thermal parameters of these configurations are obtained (Table 5. 


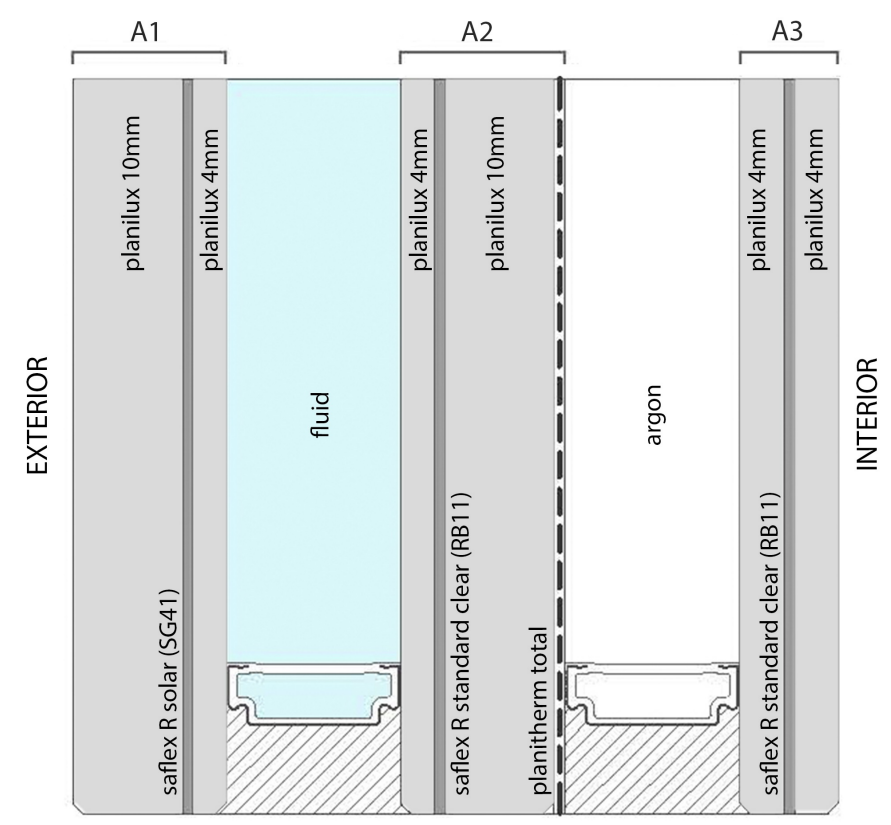

Figure 7: Triple glazing with an air chamber facing indoors and a water chamber facing outdoors configuration.

To explain in greater detail the different configurations, Figures 6 8 show the glass and PVB layers position. It is important to note that each glass pane is laminated with glass and PVB layers. Since the proposed model of this work considers an unique absorption factor for each glass pane and taking into account the hypothesis of section 3 , the absorptance of this equivalent glass pane is the sum of the absorptances of each layer which composes the glass pane. The absorptances of the equivalent glass panes are those shown in Table 4 ,

Comparing the results for the first three configurations in Table 5 the following conclusions can be drawn. The highest range of values of the $g$ factor is given in the glazing with an air chamber and a water chamber facing indoors. It may be a striking result considering equation (58), because this glazing has the lowest net absorptance of the water chamber $A_{v}$. This fact can be explained since, even it has the lowest $A_{v}$ value, $U_{i}$ is much higher than $U_{e}$.

Concerning $A_{v}$, as stated above, the glazing of Figure 8 presents the lowest value. The main reason is that the water chamber is facing indoors and, consequently, it receives less solar energy because it is dissipated in previous layers. Looking at Table 4 the absorptance of the glass pane situated just before the water chamber and the absorptance of the water chamber itself are lower in the glazing with an air chamber and a water chamber facing indoors than in the other two configurations. Concerning the energy which is directly transferred indoors, the glazing with an air chamber and a water chamber facing outdoors has the highest $A_{i}$ value, which is harmful as it has been previously explained.

Moreover, the thermal transmittance (water chamber-interior) with high flow rate, $U_{w}^{O N}$, is the capacity that the glazing has to create a thermal envelope. Therefore, Table 5 shows that the glazing with an 


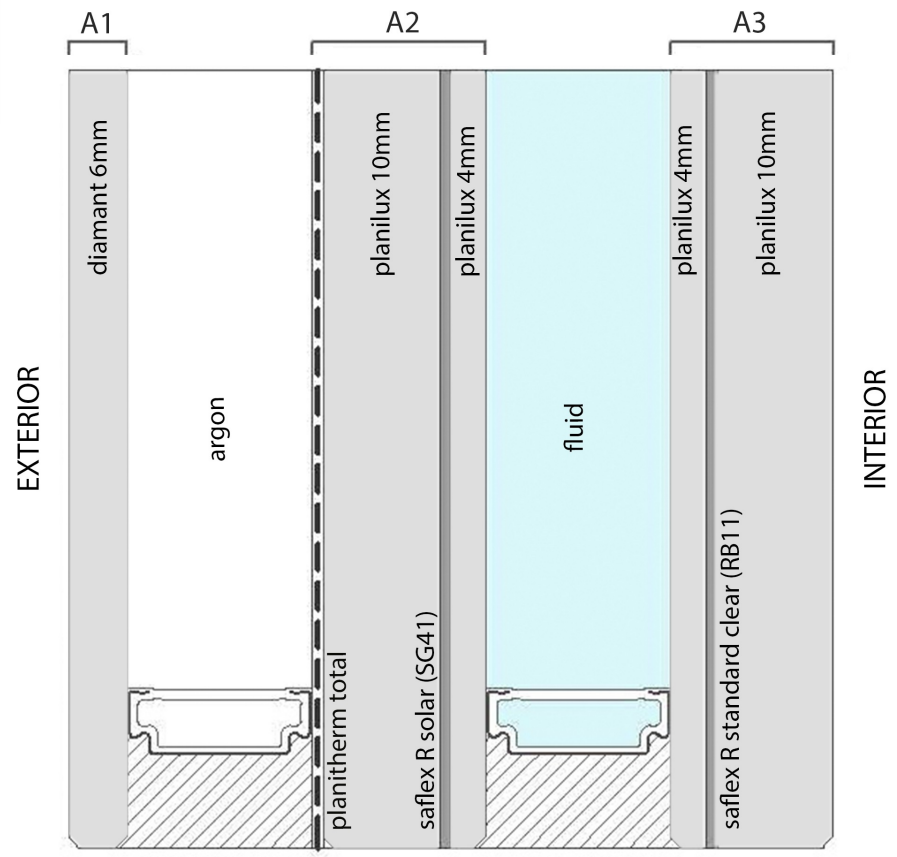

Figure 8: Triple glazing with an air chamber facing indoors and a water chamber facing indoors configuration (case 1).

air chamber and a water chamber facing outdoors does not have almost this capability. To end with the analysis of the first three configurations, the glazing with only a water chamber has a very low thermal resistance because its $U^{O F F}$ value is too high. The thermal transmittance of the other configurations are equal since their layers are the same but in a different order.

Table 5 shows two extra examples focused on the glazing configuration selected in section 7 , which is the best one. Case 2 worsens the thermal performances in comparison with the first case, because $A_{3}$ has risen at the expense of $A_{2}$. Hence, $A_{v}$ is lower and $A_{i}$ is bigger because the energy absorbed in the third glass pane goes easily indoors, meanwhile the thermal resistance of the air chamber facilitates energy transfer from the second glass pane to the water chamber. This case enlightens the discussion done at the end of section 9.1 to maximize $A_{v}$ by choosing the spectral absorptance of each layer. There may also be appreciate the slight $g^{O F F}$ fall due to the $A_{v}$ fall.

The last example shown in Table 5 does not report a big difference with case 1. Even though the small decline in performance seen in a smaller range of the values of the $g$ factor compared with case 1 , this glazing has a slightly higher transmittance, which leads to an increased day lighting. From this case, it can therefore be concluded that it is not worth the effort needed to investigate the NIR additives because solar PVB is good enough. The only justification for it would be seeking for a natural sunlight increase. To end with this section, it is important to set a criterion to know when the condition $\dot{m} c \gg U_{e}+U_{i}$ is fulfilled. This condition establishes the range of validity for the $O N$ values. By using the thermal parameters detailed above, this condition becomes: $\dot{m} \gg 0.0073 \mathrm{~kg} / \mathrm{m}^{2} \mathrm{~s}$ for a glazing with only a water chamber, $\dot{m} \gg 0.0055 \mathrm{~kg} / \mathrm{m}^{2} s$ for a glazing with an air chamber and a water chamber facing outdoors, 


\begin{tabular}{|c|c|c|c|c|c|}
\hline Glazing & $A_{1}$ & $A_{2}$ & $A_{3}$ & $A_{w}$ & $T$ \\
\hline Water chamber & 0.585 & 0.037 & - & 0.014 & 0.262 \\
\hline Water chamber facing outdoors & 0.591 & 0.055 & 0.015 & 0.014 & 0.206 \\
\hline Water chamber facing indoors (case 1) & 0.038 & 0.458 & 0.031 & 0.007 & 0.232 \\
\hline Water chamber facing indoors (case 2) & 0.038 & 0.246 & 0.187 & 0.049 & 0.232 \\
\hline Water chamber facing indoors (case 3) & 0.038 & 0.245 & 0.035 & 0.152 & 0.278 \\
\hline
\end{tabular}

Table 4: Absorptances of each layer and transmittance for different water flow glazings.

\begin{tabular}{|c|c|c|c|c|c|c|}
\hline Glazing & $A_{v}$ & $A_{i}$ & $g^{O N}$ & $g^{O F F}$ & $U_{w}^{O N}\left(\frac{W}{m^{2} K}\right)$ & $U^{O F F}\left(\frac{W}{m^{2} K}\right)$ \\
\hline Water chamber & 0.524 & 0.003 & 0.265 & 0.413 & 7.407 & 5.306 \\
\hline Water chamber facing outdoors & 0.551 & 0.014 & 0.220 & 0.248 & 1.003 & 0.952 \\
\hline Water chamber facing indoors (case 1) & 0.491 & 0.002 & 0.234 & 0.662 & 7.407 & 0.952 \\
\hline Water chamber facing indoors (case 2) & 0.467 & 0.014 & 0.246 & 0.653 & 7.407 & 0.952 \\
\hline Water chamber facing indoors (case 3) & 0.429 & 0.003 & 0.281 & 0.654 & 7.407 & 0.952 \\
\hline
\end{tabular}

Table 5: Thermal performances of different water flow glazings.

565 values can be converted to a more widely used measurement units, becoming a flow rate much larger than $0.4 \mathrm{l} / \mathrm{m}^{2} \mathrm{~min}, 0.3 \mathrm{l} / \mathrm{m}^{2} \mathrm{~min}$ and $0.1 \mathrm{l} / \mathrm{m}^{2} \mathrm{~min}$ respectively.

\section{Validation of thermal performances}

In this section various examples are included in order to validate the model presented. The validation was accomplished using Optics and Window (Finlayson et al. 1993) to determine spectral properties which are parameters for the proposed model. To validate the proposed model, water flow glazing performances given in Chow et al. (2011b) are used.

The configuration defined in Chow et al. (2011b) comprises an absorptive glass pane of $6 \mathrm{~mm}$, a water chamber of $10 \mathrm{~mm}$ and clear glass pane of $6 \mathrm{~mm}$. In this paper the thermal performances of this glazing 
are evaluated. Namely, the room heat gain and the water heat gain are discussed as functions of solar irradiance, outdoor and indoor temperatures. In our work, the room heat gain is denoted by $q$ and the water heat gain is denoted by $P$.

To validate the performances of the proposed model with the results obtained in Chow et al. (2011b), the spectral and thermal parameters used by expressions 38 and 48 should be given. Regarding the spectral parameters, Table 6 shows the absorptances obtained from Optics and Windows for the configuration given in Chow et al. (2011b). The procedure for obtaining the absorptances is the same as that followed in section 12 .

\begin{tabular}{|c|c|c|c|}
\hline$A_{1}$ & $A_{w}$ & $A_{2}$ & $T$ \\
\hline 0.511 & 0.087 & 0.047 & 0.251 \\
\hline
\end{tabular}

Table 6: Absorptances of each layer and transmittance of the water flow glazing for validation.

Regarding the thermal parameters, the coefficients $h_{i}$ and $h_{e}$ are taken from the European Standard (EN 673 , 2011), being $h_{i}=8 \mathrm{~W} / \mathrm{m}^{2} \mathrm{~K}$ and $h_{e}=23 \mathrm{~W} / \mathrm{m}^{2} \mathrm{~K}$ for common vertical glazings. The value of $h_{w}$ is calculated from the Nusselt number of 7.541 given in equation (11) of Chow et al. (2011b), considering a water chamber of $10 \mathrm{~mm}$ and $0.6 \mathrm{~W} / \mathrm{mK}$ as the thermal conductivity of the water. With these values, $h_{w}=452 \mathrm{~W} / \mathrm{m}^{2} \mathrm{~K}$.

To compare the proposed model with the thermal performances results of Chow et al. (2011b) the same boundary conditions are considered. The mass flow rate per unit of surface used by Chow et al. (2011b) is $\dot{m}=0.8 \mathrm{~kg} / \mathrm{m}^{2} \mathrm{~s}$. Besides, the inlet temperature of the water chamber was taken the same as the outdoor temperature.

The results presented in Chow et al. (2011b) are obtained for a vertical glazing with two different levels of global solar irradiance: $1000 \mathrm{~W} / \mathrm{m}^{2}, 300 \mathrm{~W} / \mathrm{m}^{2}$ with an incident angle of $60^{\circ}$. The normal incident ¿solar irradiance $\left(i_{0}\right)$ for these levels of global solar irradiance has been obtained from Figure 2 of Chow et al. (2011b), with an incident angle of $60^{\circ}$. In Figures 912 , solid lines and dashed lines are for indoor air temperature at $25^{\circ} \mathrm{C}$ and $21^{\circ} \mathrm{C}$ respectively.

Room heat gain $q$ given by expression 38 is plotted in Figure 9 together with the results obtained in Chow et al. (2011b). With the spectral and thermal parameters given above, the error of the proposed model is less than $6 \%$. The same comparison is presented in Figure 11 for the water heat gain given by expression (48). In this case, the error is less than 15\%. Since the model presented in Chow et al. (2011b) is non-linear, the thermal parameters depend on the temperatures of the solution and the accuracy of the model is higher. The main source of error is due to a non accurate prediction of the spectral parameters of the glazing used by Chow et al. (2011b). Therefore, in Figures 10 and 12 the same comparison is plotted for the room heat gain and the water heat gain by modifying slightly the spectral parameters: the thermal transmittance $T$ has been changed from 0.251 to 0.24 and the absorptance $A_{1}$ from 0.511 to 
0.4. Regarding the thermal parameters, the convective coefficient $h_{i}$ has been modified from $8 \mathrm{~W} / \mathrm{m}^{2} \mathrm{~K}$ to $8.5 \mathrm{~W} / \mathrm{m}^{2} \mathrm{~K}$. The results presented in these two graphs present an error less than $2.5 \%$ in the case of the room heat gain and less than $5 \%$ in the case of the water heat gain.

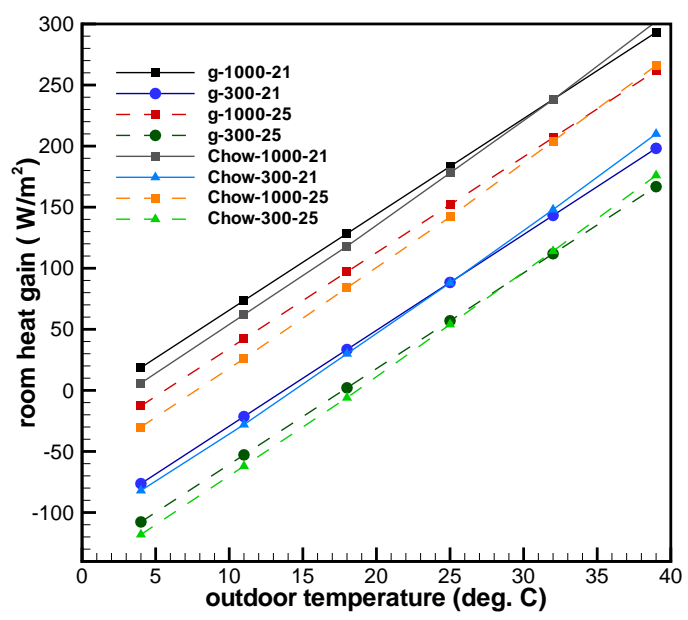

Figure 9: Performance of water flow glazing. Room heat gain a as function of the outdoor temperature for different normal incident irradiances and indoor temperatures. Comparison between results given in Chow et al. (2011b) and those calculated with the proposed model ( $g$ label) with standard parameters.

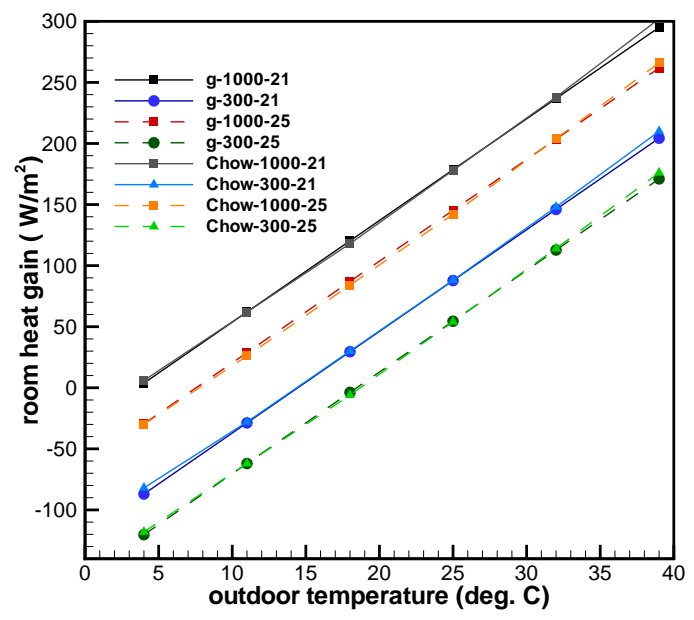

Figure 10: Performance of water flow glazing. Room heat gain a as function of the outdoor temperature for different normal incident irradiances and indoor temperatures. Comparison between results given in Chow et al. (2011b) and those calculated with the proposed model ( $g$ label) with adjusted parameters. 


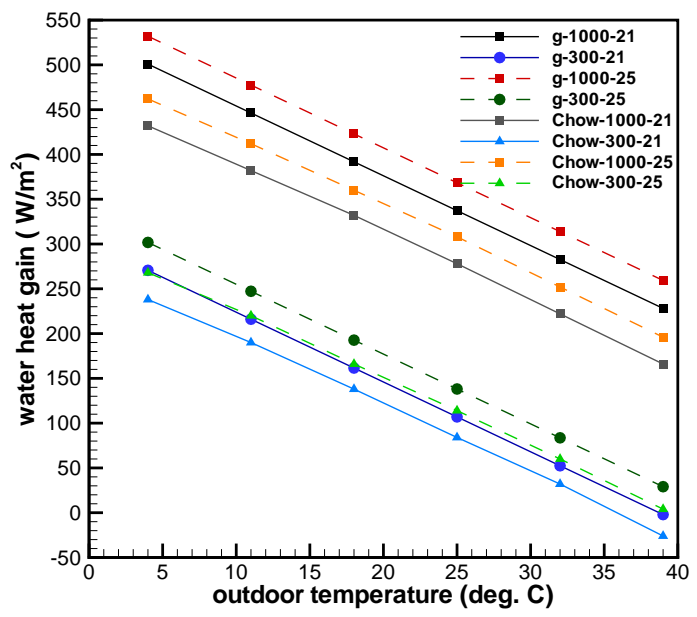

Figure 11: Performance of water flow glazing. Water heat gain versus outdoor temperature for different normal incident irradiances and indoor temperatures. Comparison between results given in Chow et al. (2011b) and those calculated with the proposed model ( $g$ label) with standard parameters.

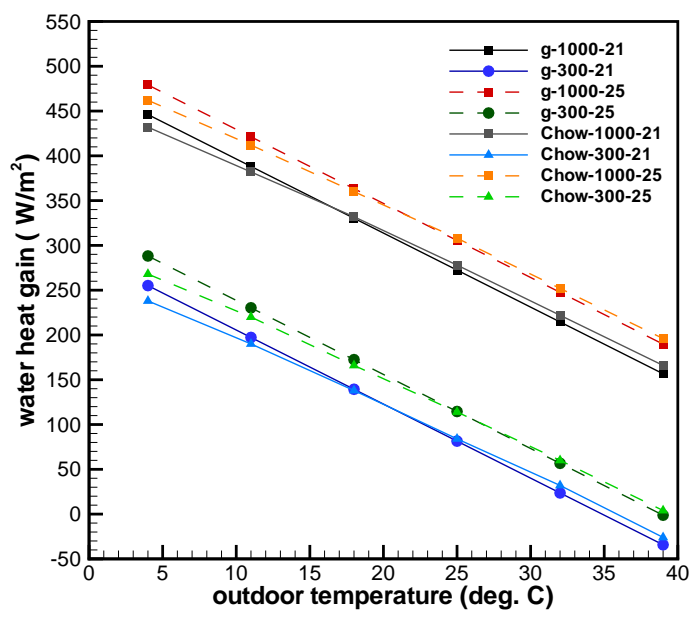

Figure 12: Performance of water flow glazing. Water heat gain versus outdoor temperature for different normal incident irradiances and indoor temperatures. Comparison between results given in Chow et al. (2011b) and those calculated with the proposed model ( $g$ label) with adjusted parameters.

\section{Conclusions}

This work shows that it is possible to determine a $g$ factor and a $U$ value for a water flow glazing which matches the classical definitions of a double glazing. Besides, it appears a second thermal transmittance $U_{w}$ associated to the water chamber. While in classical double glazing these values are constants, in water flow glazing these values can be actively controlled or modified by the flow rate $\dot{m}$.

To summarize this analysis, the following conclusions can be derived: 


\section{Acknowledgements}

The authors would like to thank Dr. Zamecnik for insightful discussions. This work was supported by program Horizon 2020-EU.3.3.1.: Reducing energy consumption and carbon footprint by smart and sustainable use, project ref. 680441 InDeWaG: Industrialized Development of Water Flow Glazing Systems. 
Baehr, H.D., Stephan, K., 2006. Heat and Mass Transfer. 2nd ed., Springer.

Bansalhail, N.K., Shail, Gaur, R.C., 1996. Application of $U$ and $g$ values for sizing passive heating concepts. Solar Energy 57, 361-373.

Carlos, J.S., Corvacho, H., 2015. Evaluation of the performance indices of a ventilated double window through experimental and analytical procedures: SHGC-values. Energy and Buildings 86, 886-897.

Chen, F., Wittkopf, S.K., Ng, P.K., Du, H., 2012. Solar heat gain coefficient measurement of semitransparent photovoltaic modules with indoor calorimetric hot box and solar simulator. Energy and Buildings 53, 74-84.

Chow, T.T., Li, C., 2013. Liquid-filled solar glazing design for buoyant water-flow. Building and Environment $60,45-55$.

Chow, T.T., Li, C., Lin, Z., 2010. Innovative solar windows for cooling-demand climate. Solar Energy Materials and Solar Cells 94, 212-220.

Chow, T.T., Li, C., Lin, Z., 2011a. The function of solar absorbing window as water-heating device. Building and Environment 46, 955-960.

Chow, T.T., Li, C., Lin, Z., 2011b. Thermal characteristics of water-flow double-pane window. International Journal of Thermal Sciences 50, 140-148.

EN 410, 2011. Glass in building. Determination of luminous and solar characteristics of glazing.

EN 673, 2011. Glass in building. Determination of thermal transmittance ( $U$ value). Calculation method.

Finlayson, E., Arasteh, D., Huizenga, C., Rubin, M., Reilly, M., 1993. WINDOW 4.0: Documentation of calculation procedures. Lawrence Berkeley Laboratory, University of California.

Freire, R.Z., Mazuroski, W., Abadie, M.O., Mendes, N., 2011. Capacitive effect on the heat transfer through building glazing systems. Applied Energy 88, 4310-4319.

Gil-Lopez, T., Gimenez-Molina, C., 2013. Influence of double glazing with a circulating water chamber on the thermal energy savings in buildings. Energy and Buildings 56, 56-65.

Gueymard, C.A., duPont, W.C., 2009. Spectral effects on the transmittance, solar heat gain, and performance rating of glazing systems. Solar Energy 83, 940-953.

Hale, G.M., Querry, M.R., 1973. Optical constants of water in the 200-nm to 200- $\mu$ m wavelength region. Applied Optics 12, 555-563.

Hermanns, M., del Ama, F., Hernández, J.A., 2012. Analytical solution to the one-dimensional nonuniform absorption of solar radiation in uncoated and coeated single glass panes. Energy and Buildings $47,561-571$. 
Ismail, K., Henríquez, J., 2003. Modeling and simulation of a simple glass window. Solar Energy Materials and Solar Cells 80, 355-374.

ISO 15099, 2003. Thermal performance of windows, doors and shading devices.

ISO 18292, 2011. Energy performance of fenestration systems for residential buildings - Calculation procedure.

ISO 9050, 2003. Glass in building - Determination of light transmittance, solar direct transmittance, total solar energy transmittance, ultraviolet transmittance and related glazing factors.

Kontoleon, K., 2015. Glazing solar heat gain analysis and optimization at varying orientations and placements in aspect of distributed radiation at the interior surfaces. Applied Energy 144, 152-164.

Kuhn, T.E., 2014. Calorimetric determination of the solar heat gain coefficient $g$ with steady-state laboratory measurements. Energy and Buildings 84, 388-402.

Li, C., Chow, T.T., 2011. Water-filled double reflective window and its year-round performance. Procedia Environmental Sciences 11, 1039-1047.

Lienhard IV, J., Lienhard V, J., 2008. A Heat Transfer Textbook. 3rd ed., Phlogiston Press.

Lyu, Y.L., Chow, T.T., 2015. Evaluation of influence of header design on water flow characteristics in window cavity with CFD. Energy Procedia 78, 97-102.

Maurer, C., Kuhn, T.E., 2012. Variable $g$ value of transparent façade collectors. Energy and Buildings $51,177-184$

Shen, C., Li, X., 2016. Solar heat gain reduction of double glazing window with cooling pipes embedded in venetian blinds by utilizing natural cooling. Energy and Buildings 112, 173-183. 